\title{
Filamentary field-aligned currents at the polar cap region during northward interplanetary magnetic field derived with the Swarm constellation
}

\author{
Hermann Lühr ${ }^{1}$, Tao Huang ${ }^{1,2}$, Simon Wing $^{3}$, Guram Kervalishvili ${ }^{1}$, Jan Rauberg $^{1}$, and Haje Korth ${ }^{3}$ \\ ${ }^{1}$ GFZ, German Research Centre for Geosciences, Section 2.3 "Earth's Magnetic Field", \\ 14473 Potsdam, Germany \\ ${ }^{2}$ Department of Space Physics, College of Electronic Information, Wuhan University, \\ 430072 Wuhan, China \\ ${ }^{3}$ The Johns Hopkins University, Applied Physics Laboratory, Laurel, MD 20723, USA
}

Correspondence to: Hermann Lühr (hluehr@gfz-potsdam.de)

Received: 3 May 2016 - Revised: 5 July 2016 - Accepted: 2 October 2016 - Published: 24 October 2016

\begin{abstract}
ESA's Swarm constellation mission makes it possible for the first time to determine field-aligned currents (FACs) in the ionosphere uniquely. In particular at high latitudes, the dual-satellite approach can reliably detect some FAC structures which are missed by the traditional singlesatellite technique. These FAC events occur preferentially poleward of the auroral oval and during times of northward interplanetary magnetic field (IMF) orientation. Most events appear on the nightside. They are not related to the typical FAC structures poleward of the cusp, commonly termed NBZ. Simultaneously observed precipitating particle spectrograms and auroral images from Defense Meteorological Satellite Program (DMSP) satellites are consistent with the detected FACs and indicate that they occur on closed field lines mostly adjacent to the auroral oval. We suggest that the FACs are associated with Sun-aligned filamentary auroral arcs. Here we introduce in an initial study features of the high-latitude FAC structures which have been observed during the early phase of the Swarm mission. A more systematic survey over longer times is required to fully characterize the so far undetected field aligned currents.
\end{abstract}

Keywords. Magnetospheric physics (auroral phenomena; current systems; energetic particles precipitating)

\section{Introduction}

The high-latitude region close to the geomagnetic pole is commonly termed "polar cap". It is defined as the region poleward of the auroral oval. Magnetic field lines extending out from this region are generally regarded open. That means they are connected on one end to the Earth and on the other to the solar wind. This magnetic field topology is the reason for specific particle signatures observed in the polar cap. The related homogenous precipitation of low-energy electrons is typically called "polar rain" (e.g., Gussenhoven and Madden, 1990). Accompanying ions are typically not observed (Newell et al., 2009). The origin of polar rain is believed to be the suprathermal solar-wind electron population (e.g., Fairfield and Scudder, 1985). Wing et al. (1996) successfully modeled the polar rain. In the polar cap region, the solar-wind ions cannot easily enter the magnetosphere, and, as a result, a parallel potential drop that retards electron entries arises in order to maintain charge quasi-neutrality. In the Wing et al. (1996, 2001) model, the solar-wind suprathermal electrons, which have high temperatures, can overcome this parallel potential to enter the magnetosphere, whereas the solar-wind thermal (core) electrons cannot.

Most of the time, field-aligned current (FAC) activity is low in the polar cap. However, when the interplanetary magnetic field (IMF) points northward, distinct FAC distributions emerge. The northward IMF $B_{z}$ (NBZ) FAC configuration is well known (e.g., Iijima et al., 1984; Araki et al., 1984). It is believed that magnetic merging between the northward IMF 
and the Earth's magnetic field at high latitudes poleward of the cusp gives rise to these current systems (e.g., Clauer and Friis-Christensen, 1988; Øieroset et al., 1997). Field-aligned current patches with opposite directions appear poleward of the cusp and are particularly prominent in the summer hemisphere (e.g., Vennerstroem et al., 2002). Special plasma convection patterns are associated with the NBZ FAC system. The typical two-cell convection changes into a four-cell convection pattern during northward IMF, and intense sunward plasma flows can be observed around the noon/midnight meridian (e.g., Burke et al., 1979; Heelis, 1984). As a consequence of that, Sun-aligned auroral arcs, also referred to as "theta aurora", appear (e.g., Frank et al., 1986). The location of highest activity in the polar cap during northward IMF depends strongly on the orientation and intensity of the other two IMF components. The IMF $B_{y}$ component causes a dawn-dusk asymmetry. In the Northern Hemisphere, for example, a positive $B_{y}$ favors the dawnside and negative $B_{y}$ the duskside. Opposite effects are observed in the Southern Hemisphere. In the case of IMF $B_{x}$ dependence, a dominant positive, Sun-pointing component supports stronger FAC intensity in the Southern Hemisphere, due to anti-parallel orientations of IMF and southern lope field lines. This favors magnetic merging in the Southern Hemisphere (e.g., Fairfield and Scudder, 1985). A dominant negative IMF $B_{x}$ is favorable for the Northern Hemisphere (e.g., Wang et al., 2014). As expected, seasonal effects also play a role. The higher conductivity during local summer supports stronger currents at high latitudes (e.g., Wang et al., 2008).

There are many studies related to polar cap electrodynamics. Most of them are based on plasma convection, particle precipitation, and/or auroral observations (e.g., Ruohoniemi and Baker, 1998; Eriksson et al., 2008; Newell et al., 2009; Frank et al., 1986; Newell and Meng, 1995). Some studies examined the large-scale field-aligned electric field in the polar cap (e.g., Wing et al., 2015; Fairfield et al., 2008). However, relatively few papers focus on observed field-aligned current distributions (e.g., Korth et al., 2004, 2005; Vennerstroem et al., 2005). This is probably due to the fact that FACs in this region have variable current geometries and therefore cannot easily be estimated from single-satellite magnetic field measurements. To our knowledge there is at present no comprehensive survey of FAC distribution in the polar cap. With the advent of ESA's constellation mission Swarm, the basis for such a study has significantly improved. By using the dual-satellite technique, the FAC density can be determined independently of its geometry. Here we present an initial study focusing on high-latitude FAC features that have been missed by single-satellite estimates. A set of welldocumented events from 2014 is presented and interpreted. This gives an idea of what a comprehensive survey may provide in future.

In the sections to follow we will first introduce the Swarm constellation and the Defense Meteorological Satellite Program (DMSP) satellites. The techniques of single- and dual-satellite FAC estimates are briefly described. Our observations of field-aligned currents in combination with particle precipitation and auroral imaging data are presented in Sect. 3. In Sect. 4, we interpret our observations and discuss them with respect to previous publications. Finally, we summarize our observations and draw conclusions in Sect. 5.

\section{Data and processing approach}

\subsection{Swarm mission and field-aligned current estimate}

ESA's Earth observation mission Swarm (Friis-Christensen et al., 2008) was launched in November 2013 into a nearpolar orbit $\left(87.4^{\circ}\right.$ inclination). The final constellation of the three-satellite mission was achieved on 17 April 2014. Since then the two satellites Swarm A and C have flown side by side, separated only by $1.4^{\circ}$ in longitude at an altitude of about $460 \mathrm{~km}$. Swarm B is cruising somewhat higher at $520 \mathrm{~km}$ altitude and will not be considered here. For this study, we make use of the magnetic field vector data, sampled by the fluxgate magnetometer (VFM). The vector data are calibrated routinely against the Absolute Scalar Magnetometer (ASM), a helium vapor instrument, for ensuring high absolute accuracy of the independent datasets from the two spacecraft. For the field-aligned current estimates presented in this study, the fully calibrated $1 \mathrm{~Hz}$ Swarm A and C vector data are used.

The vertical current density, $j_{z}$, is derived by applying Ampère's law in integral form to the vector magnetic field data,

$j_{z}=\frac{1}{\mu_{0} A} \oint \boldsymbol{B}_{\mathrm{H}} \cdot \mathrm{d} \boldsymbol{l}$,

where $A$ is the area encircled by the contour, $\boldsymbol{B}_{\mathrm{H}}$ is the magnetic field in the horizontal plane generated by the current, $\mathrm{d} \boldsymbol{l}$ is the line element along the integration path, and $\mu_{0}$ is the permeability of free space. In practice, we apply a discrete form for solving the integral along straight-line elements between four corners forming a quadrangle. To first order, our analysis returns the average current density flowing through the encircled area. Two sides of the considered quadrangle lie on the Swarm A and C tracks representing the along-track gradient between two measurement points $5 \mathrm{~s}$ apart. This corresponds to a distance of $38 \mathrm{~km}$ due to the orbital velocity of $7.6 \mathrm{~km} \mathrm{~s}^{-1}$. The remaining two sides in cross-track direction connect measurement points of the two satellites located at the same magnetic latitudes. Since the orbital planes are separated by $1.4^{\circ}$ in longitude, the distance changes with latitude. At geographic latitudes above $86^{\circ}$, no current densities are calculated because the spacecraft separation near the crossover point at $87.3^{\circ}$ latitude is too small. More details about the Swarm current density estimation have been presented elsewhere (Ritter et al., 2013; Lühr et al., 2015b).

For our analysis, the magnetic effect caused by the currents is of interest. Therefore, appropriate models for the 
core, crustal, and large-scale magnetospheric magnetic fields (in this case the CHAOS-5; Finley et al., 2015) have been subtracted from the measurements. In a next step, the field residuals are high-pass-filtered with a $3 \mathrm{~dB}$ cutoff period of $20 \mathrm{~s}$ (corresponding to an along-track wavelength of $150 \mathrm{~km}$ ) to eliminate the influence of small-scale FACs, which may cause spatial aliasing between the readings at the four corners. Furthermore, Lühr et al. (2015a) showed that largescale FACs $(>150 \mathrm{~km})$ are spatial structures generally stationary for more than $1 \mathrm{~min}$. Estimates of FAC density are calculated for every second, providing a value every $7.6 \mathrm{~km}$, but the applied $20 \mathrm{~s}$ filter suppresses FAC structures smaller than $150 \mathrm{~km}$.

For obtaining field-aligned currents estimates, the vertical currents are mapped onto the main field direction by considering the magnetic inclination, $I$ :

$j_{\|}=\frac{j_{z}}{\sin I}$.

For completeness, FAC estimates from the Swarm A and C spacecraft, based on the single-satellite approach, are also derived. Here the standard processing approach is applied,

$j_{\|}=\frac{1}{\mu_{0} v} \frac{\Delta B_{y}}{\Delta t}$,

where $B_{y}$ is the residual horizontal magnetic field component perpendicular to the orbit track, $\Delta t$ is in our case a time step of $1 \mathrm{~s}$, and $v$ is the satellite velocity component perpendicular to the main field direction. For a direct comparison with the above-described dual-satellite FACs, the data are also filtered by the same $20 \mathrm{~s}$ low pass. This simple current-estimate approach depends on the validity of some assumptions such as the stationarity of the current system and on the organization of FACs in sheets oriented perpendicular to the orbit track. More details on the validity of these assumptions can be found in Lühr et al. (2015a).

\subsection{The Defense Meteorological Satellite Program (DMSP) mission}

The DMSP is a long-term operational meteorological, oceanographic, and solar-terrestrial physics program of the US Department of Defense. Several satellites have been maintained in orbit since the first launch in 1962 (then known as Program 35), and the data have been released publicly for scientific studies since 1972. The spacecraft are on circular, Sun-synchronous orbits ( $98^{\circ}$ inclination) at $840 \mathrm{~km}$ altitude and an orbit period of approximately $101 \mathrm{~min}$. Most of them circle the Earth near the dawn-dusk plane. On all recent DMSP satellites the SSJ4/5 instrument package includes the curved plate electrostatic analyzers to measure ions and electrons from $32 \mathrm{eV}$ to $30 \mathrm{keV}$ in logarithmically spaced steps (Hardy et al., 1984). Because of its upward-pointing and limited pitch-angle resolution, DMSP SSJ4/5 measures only highly field-aligned precipitating particles. Since 2003
DMSP satellites have also carried the Special Sensor Ultraviolet Scanning Imager (SSUSI) with a global far-ultraviolet (FUV) auroral imager (Paxton et al., 2002). For this study, we make use of the observations from these instruments on the satellites F16, F17, and F18.

\subsection{Solar-wind and interplanetary magnetic field data}

For characterizing the solar-wind input to the magnetosphere, OMNI data are used. OMNI is a multi-source dataset from different spacecraft (e.g., ACE, WIND, IMP-8) assembled by the National Space Science Data Center (NSSDC). Data resolution ranges between 1 min and 27 days. They are publicly available at the Space Physics Data Facility (SPDF) of NASA's Goddard Space Flight Center (http://omniweb. gsfc.nasa.gov). High-resolution (1 min) OMNI solar-wind and IMF data, which have been time-propagated to the Earth's bow shock, are used here. We have further averaged the data to $15 \mathrm{~min}$ periods. Prevailing IMF conditions around the epochs of our high-latitude FAC events have been listed in Table 1. An expected delay of $25 \mathrm{~min}$ for the signal propagation from the bow shock to the ionosphere is taken into account. Delay times of the order of 20 min have been derived in earlier studies for the dayside (e.g., Vennerstroem et al., 2002; He et al., 2014). On the nightside even longer delay times of order $1 \mathrm{~h}$ have been reported (e.g., He et al., 2014, and references therein).

\section{Observations}

For this initial study we consider the time period from middle of April 2014, when the Swarm orbit constellation was achieved, to the end of 2014. For this time interval, the mostprecise FAC estimates are available because ASM data are available for calibrating the vector field measurements of both Swarm A and C. We make use of the Swarm level 2 data product "Field-Aligned Current", which is publicly available at https://earth.esa.int/web/guest/swarm/data-access.

\subsection{Field-aligned currents at high latitude}

The Swarm constellation mission allows for the application of various techniques for estimating FAC densities from near-simultaneous magnetic field readings of one, two, or three spacecraft (e.g., Ritter et al., 2013; Dunlop et al., 2015). For this study, we focus on dual-spacecraft FAC estimates from the side-by-side pair Swarm A/C as described in Sect. 2 but also present some single-satellite results for comparison.

Figure 1 shows the three independent FAC estimates for one pass over the northern auroral region. Here the two FAC curves from the single satellites (red and blue) agree very well with the dual-satellite results (black curve). Please note that positive values in the Northern Hemisphere reflect downward currents and in the Southern Hemisphere positive currents flow upward, just following the direction of the geo- 
Table 1. Summary of locations and IMF conditions during the high-latitude FAC events.

\begin{tabular}{|c|c|c|c|c|c|c|c|}
\hline \multirow[t]{2}{*}{ Event } & \multirow[t]{2}{*}{ UT } & \multicolumn{2}{|c|}{ Location } & \multicolumn{3}{|c|}{ IMF } & \multirow{2}{*}{$\begin{array}{l}\text { FAC density } \\
\qquad\left(\mu \mathrm{A} \mathrm{m}^{-2}\right)\end{array}$} \\
\hline & & MLat $\left(^{\circ}\right)$ & $\operatorname{MLT}\left({ }^{\circ}\right)$ & $B_{x}(\mathrm{nT})$ & $B_{y}(\mathrm{nT})$ & $B_{z}(\mathrm{nT})$ & \\
\hline 4 Sep 2014 & $13: 53$ & -75.00 & 4.98 & -3.75 & 0.99 & 1.87 & 0.8 \\
\hline 30 Aug 2014 & $14: 55$ & -77.41 & 5.39 & 1.20 & 2.16 & 5.10 & -0.7 \\
\hline 15 Sep 2014 & $22: 17$ & -75.01 & 20.50 & 3.24 & -2.05 & 2.74 & 1.1 \\
\hline 23 Dec 2014 & $11: 02$ & 78.68 & 15.55 & 5.34 & -7.50 & 2.89 & 3.1 \\
\hline 23 Dec 2014 & $12: 36$ & 78.30 & 16.05 & 5.17 & -21.26 & 19.71 & -10.0 \\
\hline 4 Dec 2014 & $11: 58$ & 77.63 & 17.07 & 7.17 & -3.21 & 1.14 & 1.2 \\
\hline
\end{tabular}
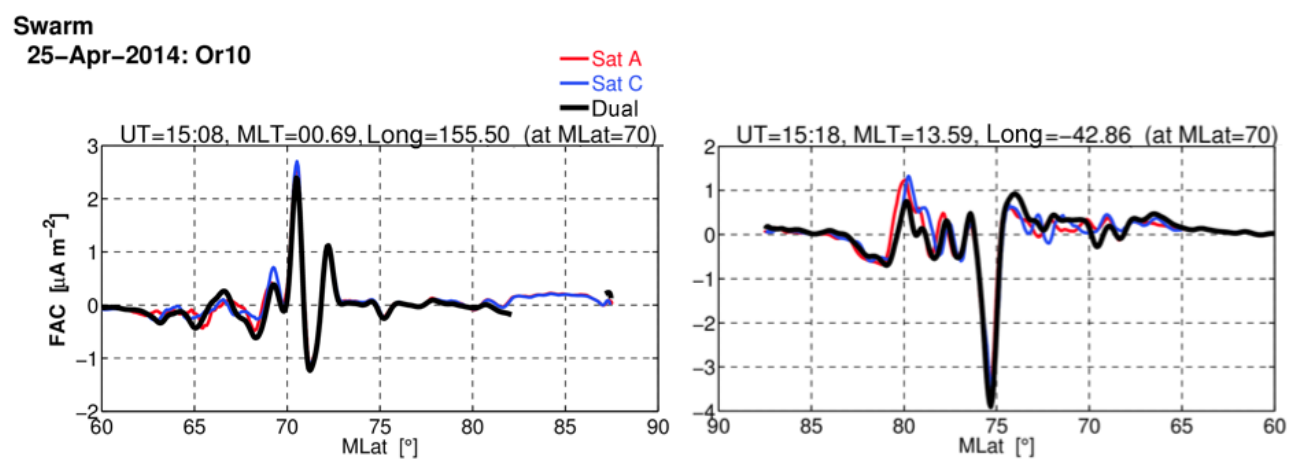

Figure 1. Example of field-aligned current recording for a high-latitude pass from post-midnight to afternoon. Generally, single- and dualsatellite FACs agree very well in the auroral region, and there is low FAC activity in the polar cap.

magnetic field. The large negative peak in the right panel of Fig. 1 can be related to upward Region 1 (R1) FACs in the afternoon sector. In the post-midnight sector (left panel) multiple FAC sheets appear, but the largest is downward, as expected for R1.

When performing systematic comparisons between singleand dual-satellite FAC results, we obtain typically a high degree of coherence at auroral latitudes (60-75 MLat). At higher magnetic latitudes (MLat), including the polar cap, clear deviations often occur. Two such examples are shown in Fig. 2. In the case of 31 May 2014 (left panel) we find a good match of all three FAC estimates up to about $75^{\circ}$ MLat, but beyond that the dual-satellite results are much more positive over a range of $5^{\circ}$ in MLat. Quite similar signatures are observed on 19 June 2014, shown in the right panel of Fig. 2. Here again the deviation of FAC estimates starts around $75^{\circ}$ MLat but continues even up to the geomagnetic pole. We regard the results based on dual-spacecraft technique as more reliable; for example, they are not dependent on the orientation of the FAC sheets. Therefore it might be interesting to identify the conditions prevailing during times of FAC mismatches.

As a first step, we look at the locations of these FAC events. Figure 3 shows the distribution of identified events poleward of $75^{\circ}$ MLat in the Northern Hemisphere for the time period April-November 2014 on mag- netic latitude versus magnetic local time (MLT) dial plots. Events adjacent to the cusp region (75-80 MLat, 10:0014:00 MLT) have been ignored. There is a clear preference for the nightside. Next we investigated possible dependences on the solar-wind and the IMF. As can be deduced from the two dial plots in Fig. 3, the polarity of IMF $B_{y}$ obviously has no significant influence on either the occurrence rate or the distribution of our FAC events. Also, the solar-wind speed and density are of no concern (not shown). In contrast, IMF $B_{z}$ obviously has a strong influence high-latitude FAC events. Figure 4 shows in the left panel, in red, the general distribution of all IMF $B_{z}$ values within our time period. The wellknown distribution emerges, centered at zero with a typical width of about $\pm 3 \mathrm{nT}$. In contrast, the IMF $B_{z}$ distribution derived for our events (in blue) is shifted by $2-3 \mathrm{nT}$ towards positive values. This indicates a clear preference of northward IMF for the cases of mismatch in FACs. The tendency becomes even clearer when looking at the IMF clock angle, $\theta=\arctan \left(B_{y} / B_{z}\right)$, (see Fig. 4 right frame). The normal distribution (in red) exhibits maxima at $\pm 90^{\circ}$ and a minimum around $0^{\circ}$. Conversely, our events show largest occurrence rates for clock angles around $0^{\circ}$. This confirms even more clearly the importance of northward orientation of the interplanetary magnetic field. 

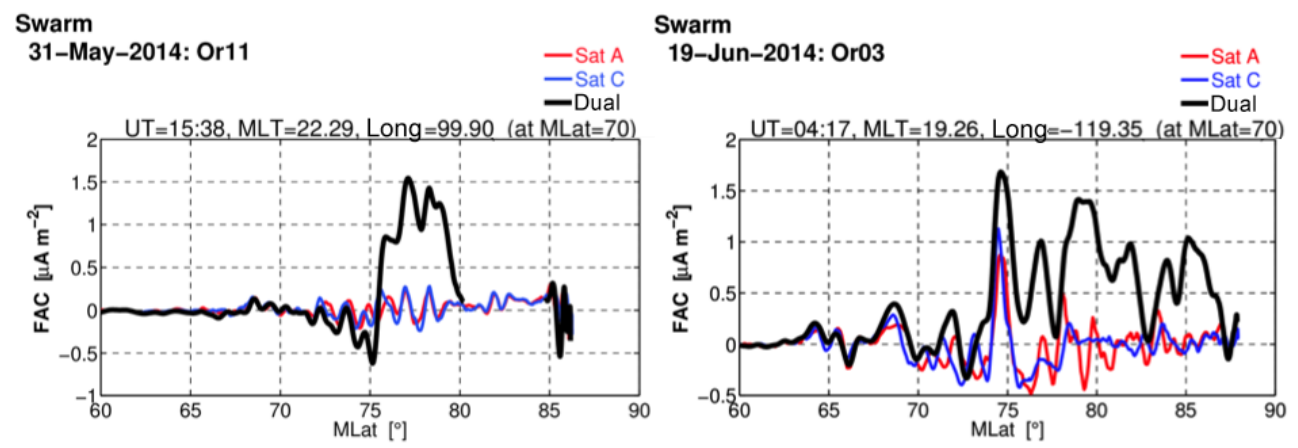

Figure 2. In the polar cap occasionally significant differences between single- and dual-satellite FAC estimates are observed.
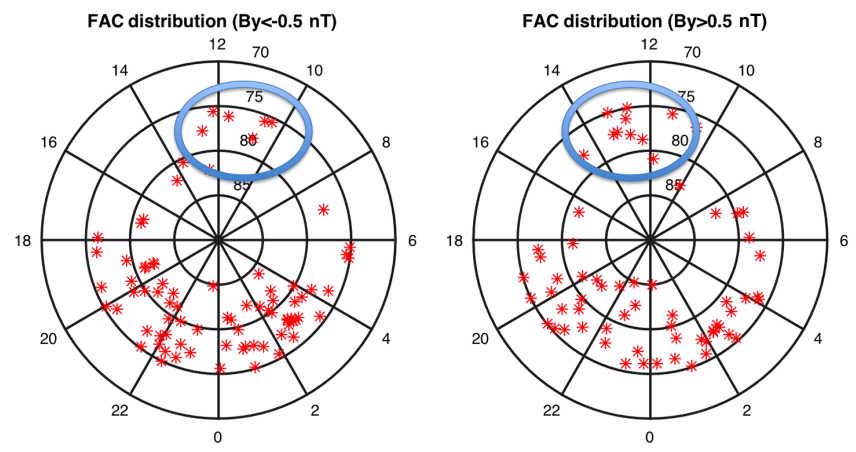

Figure 3. Distribution of polar cap FAC events showing significant difference between single- and dual-satellite estimates. Events in the cusp region (encircled) are not considered in the following. Cases are sorted by the prevailing sign of the IMF $B_{y}$ component.

\subsection{Complementary observations during events}

We are interested in discovering the features of those FACs at high latitudes which have been missed by the single-satellite approach. What is most valuable for a comparison is simultaneous particle precipitation data and, if available, auroral images. Both these types of measurements are performed by the DMSP spacecraft. The difficulty, however, is finding simultaneous and collocated measurements of Swarm and DMSP for the epochs of our events. Most of the DMSP satellites are on Sun-synchronous orbits within the dawn/dusk sector. Therefore, they pass auroral latitudes in the Northern Hemisphere preferably on the dayside (cusp region). Better chances for nightside conjunctions with Swarm are therefore expected in the Southern Hemisphere.

For our period of interest, we have identified three events in the Southern Hemisphere and also three events in the Northern Hemisphere with good data coverage. Prevailing IMF conditions for the six examples are presented in Table 1. Note that the events all occurred during northward IMF. Figure 5 shows combined data from DMSP and Swarm for the 4 September 2014 event. The closest approach occurs at $75^{\circ}$ MLat in the morning sector around 13:50UT. On the left side, the Swarm track is over-plotted on the auroral image obtained by DMSP. The location of the closest approach is marked by a red star on the track. Swarm comes from the post-midnight sector and passes over to noon. The FAC estimates are shown in the top panel. From the curve, we have identified R2 and R1 FAC regions. Poleward of these current signatures, further FAC structures appear. From the auroral image, it is obvious that there are narrow streaks of auroral forms in the morning sector reaching quite high magnetic latitudes. Swarm traverses parallel to these auroral features inside of the oval. It is therefore not surprising that singlesatellite FAC estimates cannot resolve these high-latitude currents correctly. The right part of the figure presents DMSP precipitating electron and ion spectrograms. For comparison, we have added a curve with Swarm FAC estimates obtained at the same magnetic latitudes. Our assignment of R2 and $\mathrm{R} 1$ sheets is reasonably well supported by the collocated particle spectra. The polar cap seems to be quite small during this event. Only poleward of $80^{\circ}$ MLat do we observe the expected low fluxes of electrons and the absence of ions. The Swarm orbit does not reach those latitudes on this orbit. Our high-latitude FAC, marked HC, is accompanied by a considerable flux of kilo-electronvolt ( $\mathrm{keV}$ ) electrons and somewhat less ion flux. The spectrograms of both species infer a location on closed field lines. The DMSP electron spectrogram shows that there are filamentary broadband and/or monoenergetic electron accelerations (e.g., Wing et al., 2013) near the poleward edge of the auroral oval, near the polar cap boundary. These electron accelerations are in the vicinity of where the dual-FAC technique does not agree with Swarm A and $\mathrm{C}$ results.

A quite similar event was observed on 30 August 2014 in the Southern Hemisphere. Figure 6 shows the Swarm and DMSP observations in the same format as Fig. 5. Swarm and DMSP enter the auroral zone from the post-midnight side. The auroral images again show narrow streaks in the morning sector reaching to high latitudes. From the FAC plot we can identify the region R2 and R1 FACs. A downward FAC region appears poleward of $\mathrm{R} 1$ and is collocated with narrow luminous arcs. The particle spectrograms in the right pan- 

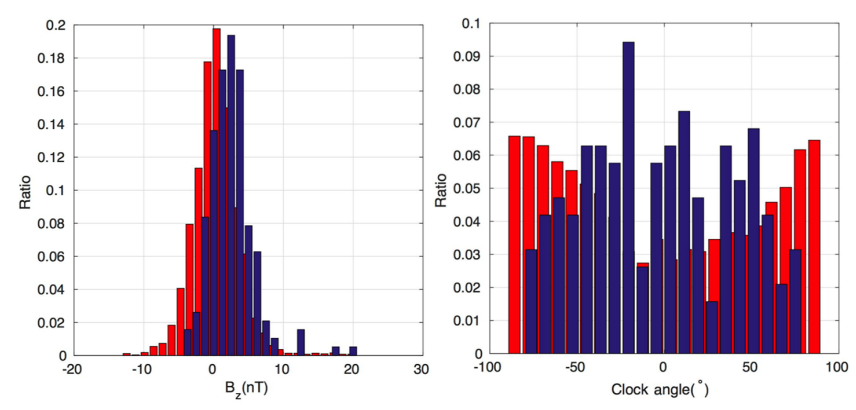

Figure 4. Occurrence distribution of interplanetary magnetic field orientations: red represents all times and blue represents polar cap FAC events. For the events there is clear a bias towards positive IMF $B_{z}$, which is confirmed by a clock angle distribution peaking around $0^{\circ}$.

els show clear signatures of filamentary accelerated electrons nearly collocated with the FAC marked HC. In addition, ion fluxes are observed at that location. All these observations confirm that our observations are on closed field lines just equatorward of the polar cap boundary. The discrepancy between single- and dual-satellite FAC estimates can again be attributed to the small angle between orbital track and auroral forms.

A third conjunction event occurred in the late evening sector (see Fig. 7). For this case, auroral images from DMSP are not available. The interesting feature here is the prominent upward FAC at high latitude. From the comparison with the DMSP particle spectrograms, we see that it appears on closed field lines near the polar cap boundary. Here again, the Swarm orbit is skimming the auroral oval. Also note that there are some structures in the precipitating electrons suggesting broadband accelerations (e.g., Wing et al., 2013).

In the Northern Hemisphere, we found events with good Swarm-DMSP conjunctions only at the end of 2014. In this hemisphere, the orientation of the auroral oval with respect to the Swarm tracks is more favorable. Of particular interest are the observations on 23 December 2014. On that day, the IMF was partly unusually strong, reaching 20 to $30 \mathrm{nT}$ with a dominant northward component (see Table 1). The observations for the first event are compiled in Fig. 8. Swarm crosses the rather contracted auroral oval first in the afternoon sector. After passing the R2 and R1 FAC sheets, an intense downward FAC is observed on the poleward side of the oval. On the nightside, Swarm crossed a fairly broad auroral region (70-80 MLat), which can be deduced both from the image and the FAC structure. The right panels of Fig. 8 show that the intense downward FACs is observed in a region of soft electron precipitation and absent of ions. This suggests a location of the FAC in the polar rain region. A minute later, auroral precipitation is observed again and drops out a little later. These observations suggest that DMSP skimmed along the poleward edge of the dayside oval. Also note that the polar rain electrons show evidence of filamentary accelerations.
A quite extreme event is observed in the next orbit, when the IMF intensity amounted to $30 \mathrm{nT}$ (see Table 1 ). As can be seen in Fig. 9, Swarm detected a very strong upward FAC of $10 \mu \mathrm{A} \mathrm{m}^{-2}$ in the afternoon sector, which we attribute to the $\mathrm{R} 1$ currents. The strong upward FAC is perhaps consistent with the increased precipitating electron flux (typically there is no one-to-one correspondence between increase/decrease in precipitating electron flux and increase/decrease in upward FAC, but in some cases where the upward FAC is strong, increased electron precipitation is usually observed). Poleward of that, an appreciable downward FAC appears. On the other side, in the post-midnight sector, Swarm passes again through a broad and active auroral region, confirmed by optical, particle precipitation, and FAC data. The comparison of FAC estimates with precipitating particles in the right panels shows that the very intense upward current is accompanied by high fluxes of soft electrons and $\mathrm{keV}$ ions. There are lots of filamentary structures in the precipitating electron data. The adjacent downward FAC is collocated with lower fluxes of particles but still on closed field lines.

Our final event, shown in Fig. 10, occurred on 4 December 2014. Here both Swarm and DMSP F17 are in dusk-todawn orbits. Swarm first crossed fairly weak FAC structures on the evening side until it reached the polar cap boundary. There discrete auroral features appear in the image, which are accompanied by prominent downward FAC sheets at 78 and $83^{\circ}$ MLat (see top left). Close to the geographic pole the dual-satellite technique is not reliable. Therefore, the $83^{\circ}$ MLat FAC peak is derived only from single-satellite solutions. On the dawnside, Swarm is passing through a wide region of auroral and FAC activity. From the comparison with the particle spectrograms (right panels) we can confirm that the downward FAC sheets at $78^{\circ}$ (marked by red dot in lower left frame) are located on closed field lines at the border to the polar cap. In the case of the FAC peak at $83^{\circ}$ MLat, Swarm and DMSP orbits are too far apart; thus, particle signature can no longer be used for direct interpretation of that sheet. However, the auroral image shows that there are filamentary structures. This event can be considered as an example for a detached Sun-aligned auroral arc.

Here the positive IMF $B_{z}$ during all events (see Table 1) should be stressed again. This is consistent with, for example, Iijima and Shibaji (1987), who stated that high-latitude FACs preferably occur during times of northward IMF.

\section{Discussion}

The Swarm constellation mission provides new opportunities for improving current density estimates in the ionosphere. This is in particular true for field-aligned currents determined from magnetic field measurements of the pair Swarm A and C, flying side by side. Systematic comparisons of FAC estimates derived in the traditional way from singlesatellite measurements with the advanced dual-satellite tech- 

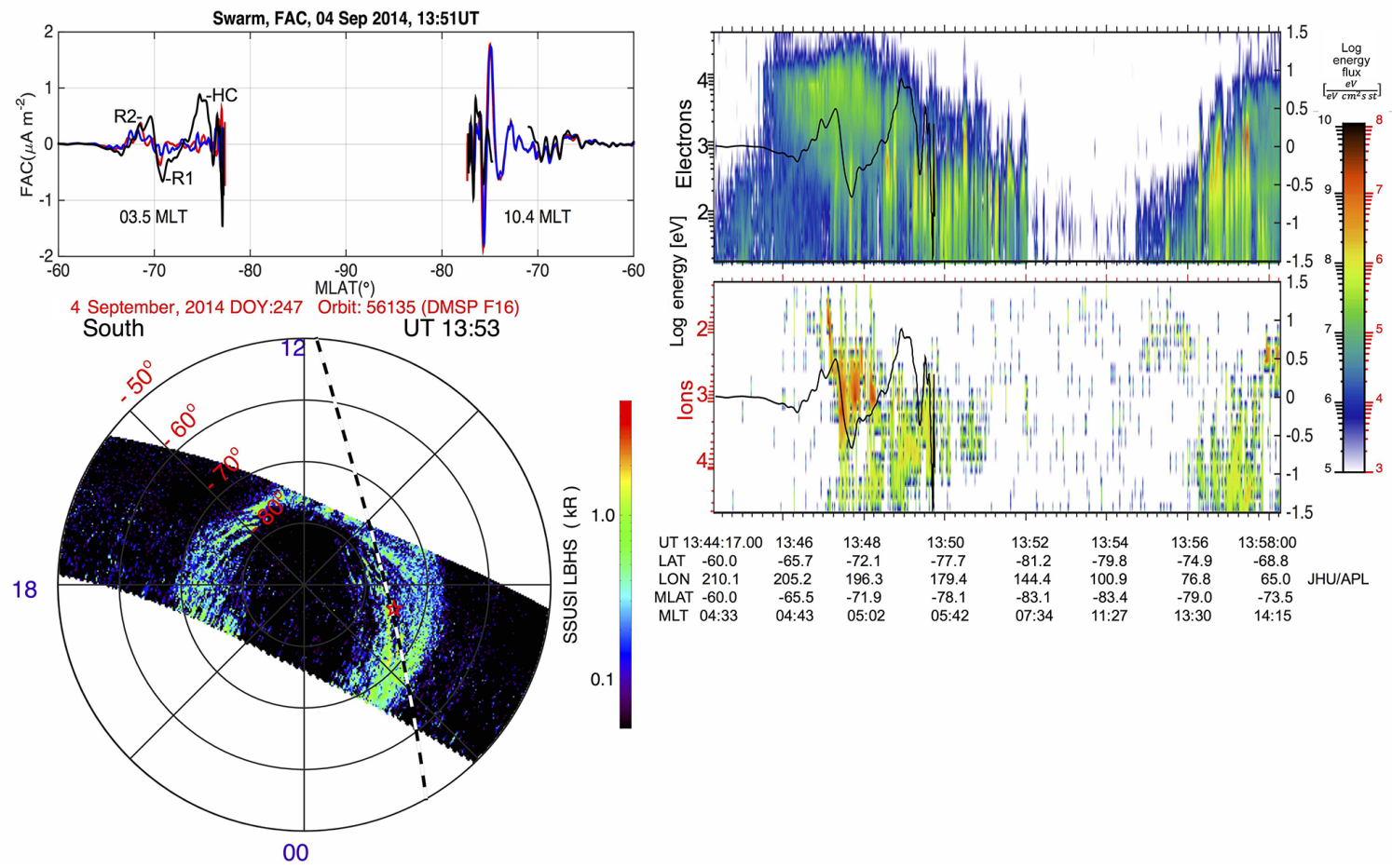

Figure 5. Composite plot of Swarm and DMSP observations of a polar cap event in the Southern Hemisphere. Presented are field-aligned currents along the Swarm track (top left), where the black curve shows dual-SC FAC and the red and blue curves show results from Swarm A and C, respectively. Auroral images and the Swarm orbit track are illustrated below. Precipitating particle spectrograms with FAC profiles over-plotted are shown to the right, with electrons at the top and ions at the bottom.
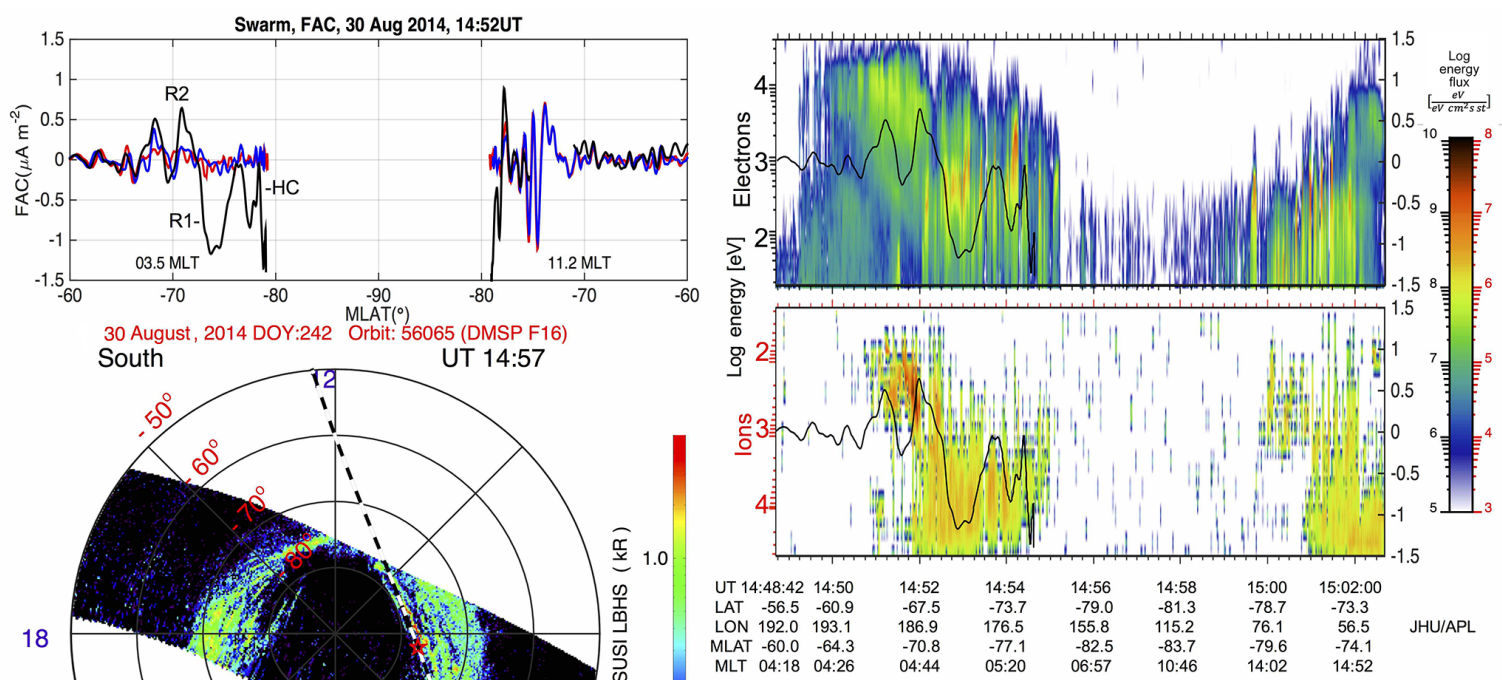

18

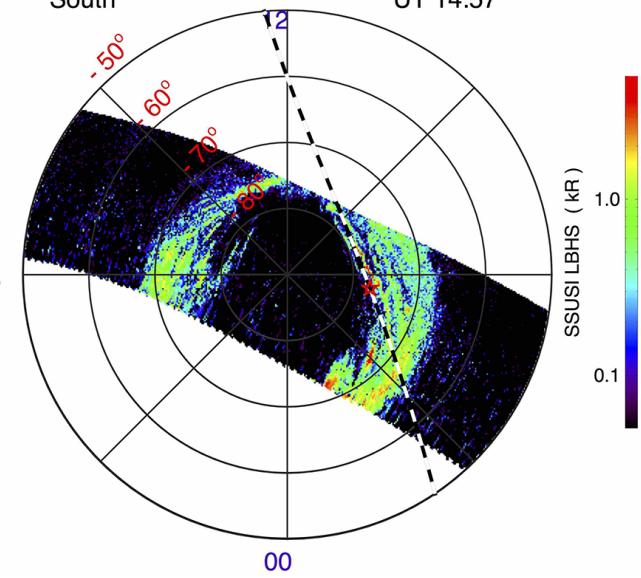

Figure 6. Same format as Fig. 5 but for 30 August 2014. 

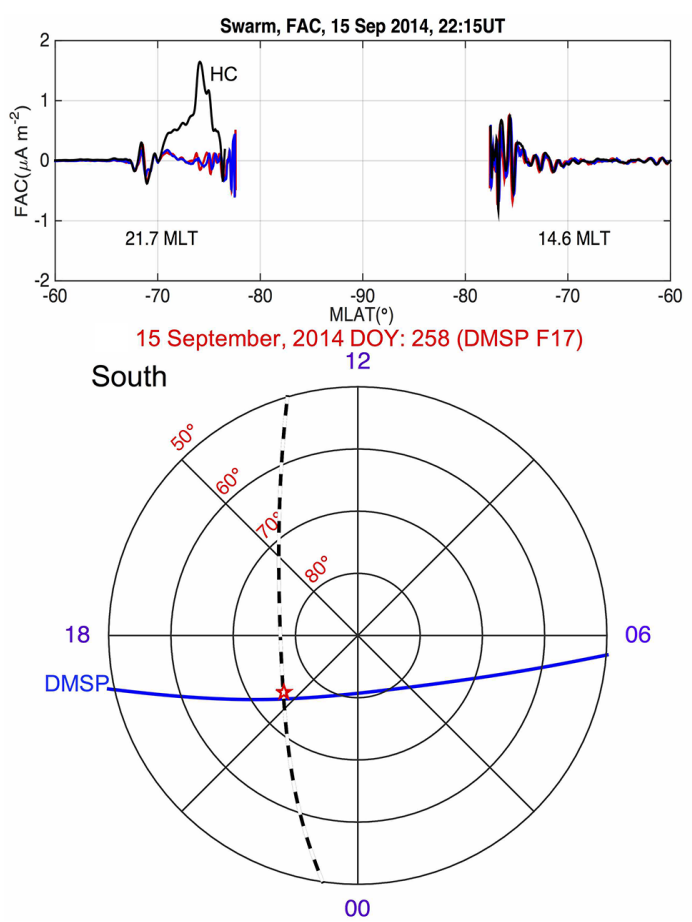

Figure 7. Same format as Fig. 5 but for 15 September 2014.

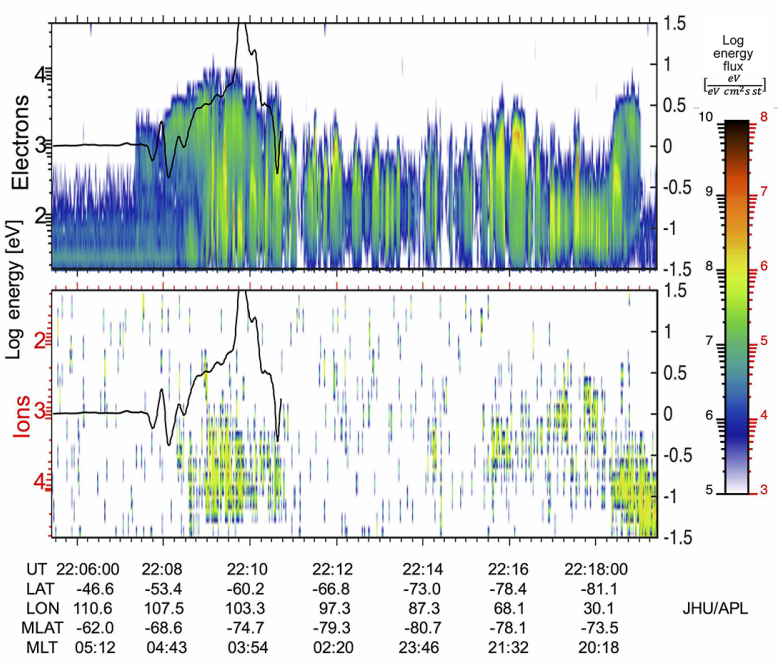

tions. However, from the limited time period of 8 months considered here, we may not be able to resolve a potential dependence on $B_{y}$. It is somewhat surprising that many more events (about 6 times more) have been found at northern high latitudes than in the Southern Hemisphere. This can partly be explained by geometric arguments. Due to the larger offset of the southern geomagnetic pole, Swarm satellites cross the polar cap only about half as frequently as in the northern geomagnetic pole. Another factor may be a seasonal bias of our study period, which preferentially covers northern summer and fall seasons. A more comprehensive survey over longer times would be needed to resolve these dependencies and to quantify a possible hemispheric imbalance.

For a more profound interpretation of the observed FAC events, concurrent observations of other quantities are of great importance. Unfortunately, there are not too many sources of supporting data. Our first choice was to examine particle precipitation fluxes accompanying our events. The six examples of concurrent particle and auroral image observations presented here (three from the Northern and three from the Southern Hemisphere) cannot be regarded as representative; rather, they simply provide an idea of some typical configurations. A rather comprehensive review of polar cap particle signatures and their relations to ambient conditions has been given by Newell et al. (2009). From particle measurements, the polar cap can be identified by the presence of polar rain. In the composite Figs. 5 to 10, we have marked FAC sheets according to the regions they are associated to. The FAC poleward of the classical R2 and R1 regions 

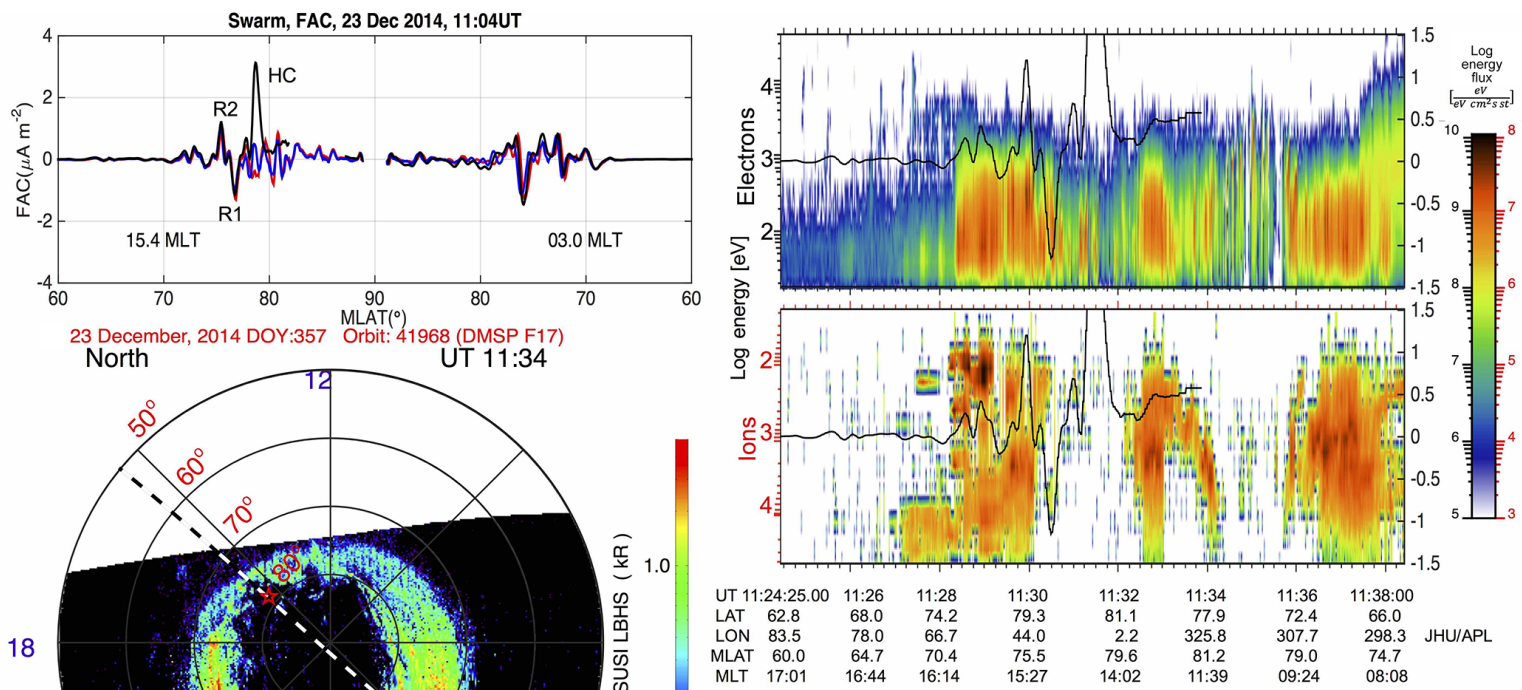

18

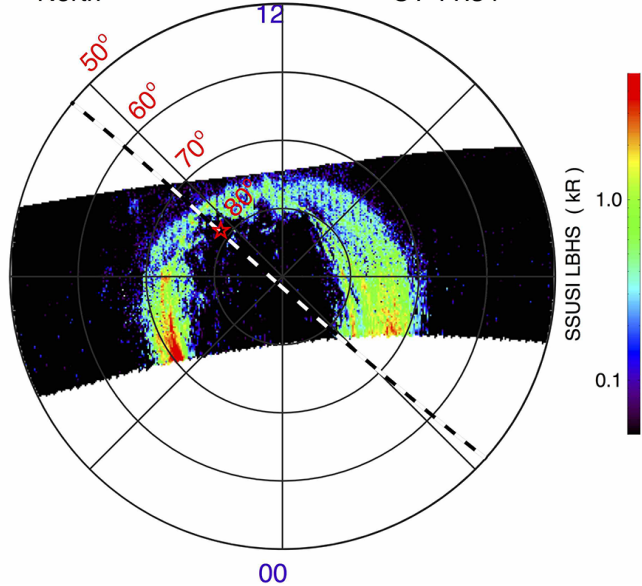
MT 17:01

Figure 8. Same format as Fig. 5 but for the Northern Hemisphere and 23 December 2014.
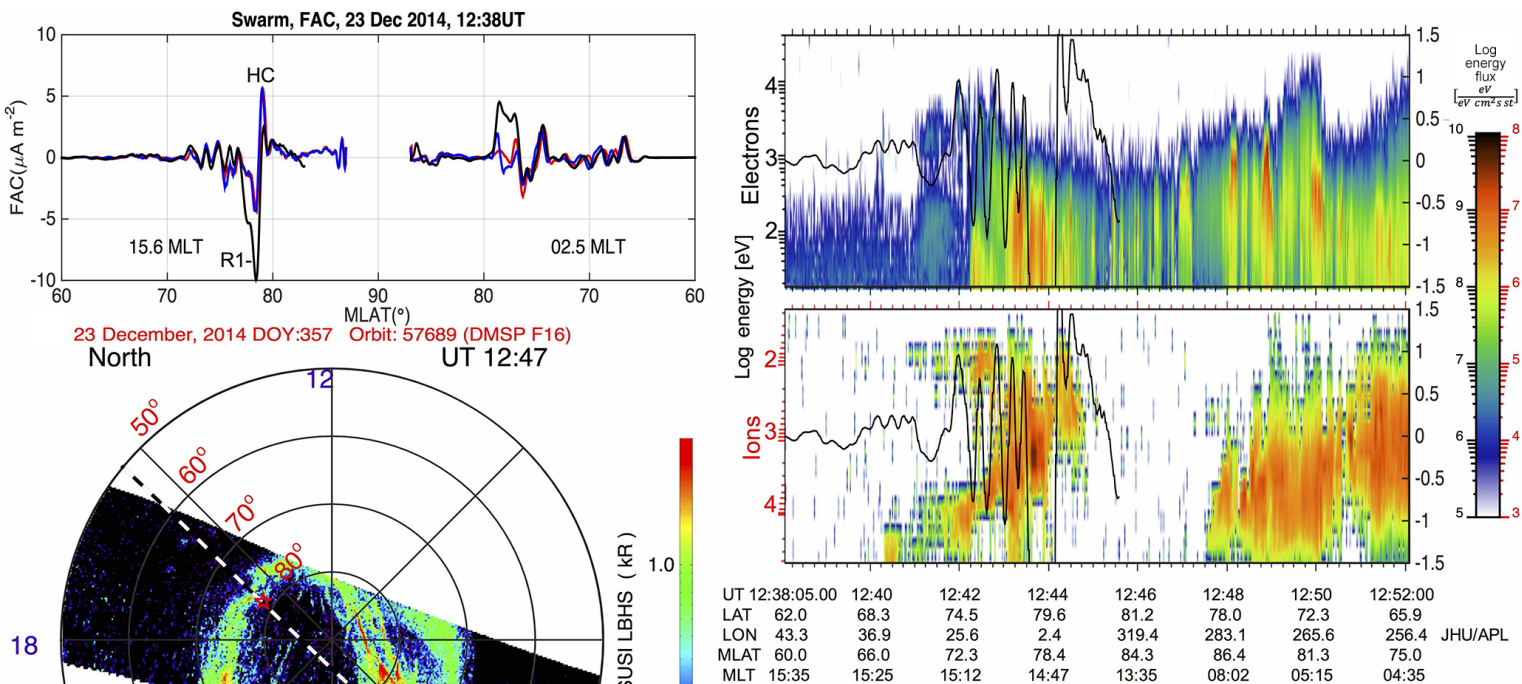

18

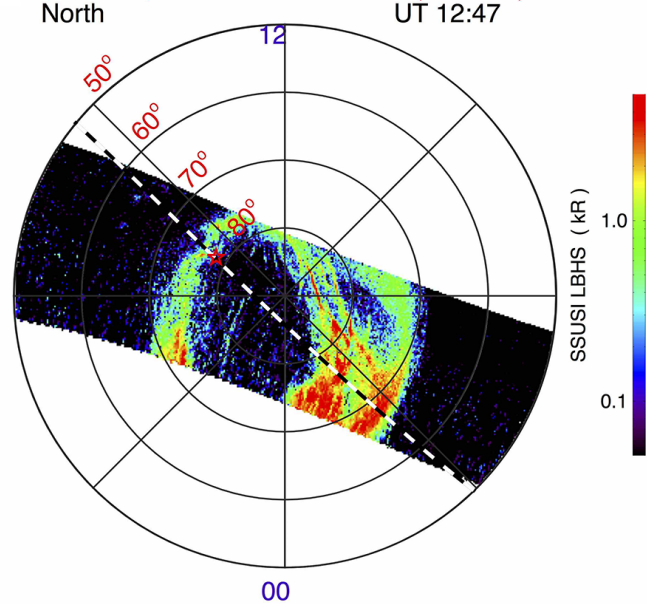

MLT 15:35 $15: 25$

Figure 9. Same as Fig. 8 but for subsequent orbit. 


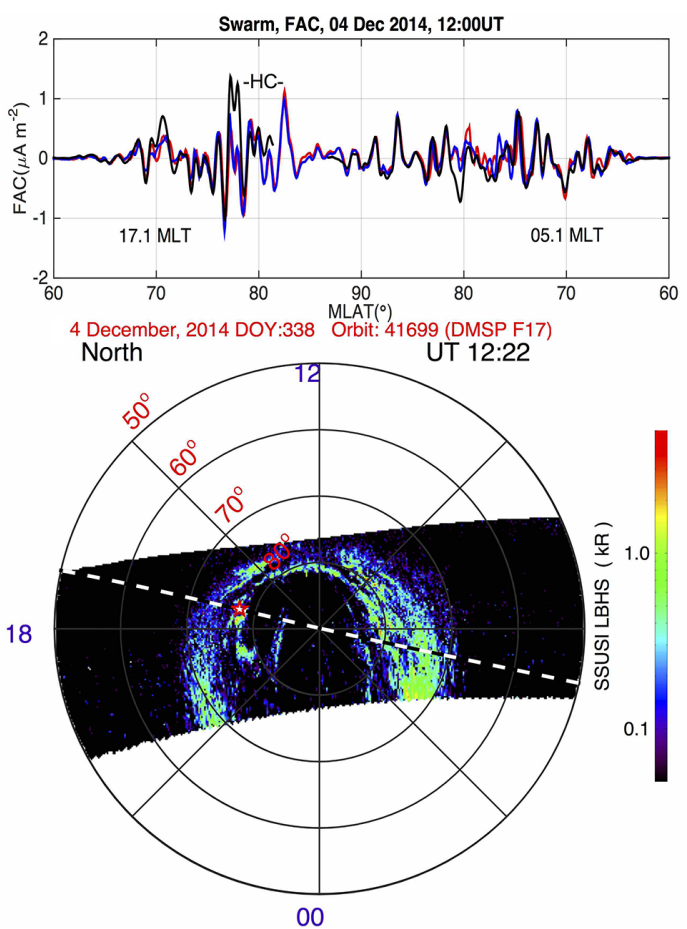

Figure 10. Same format as Fig. 8 but for 4 December 2014.

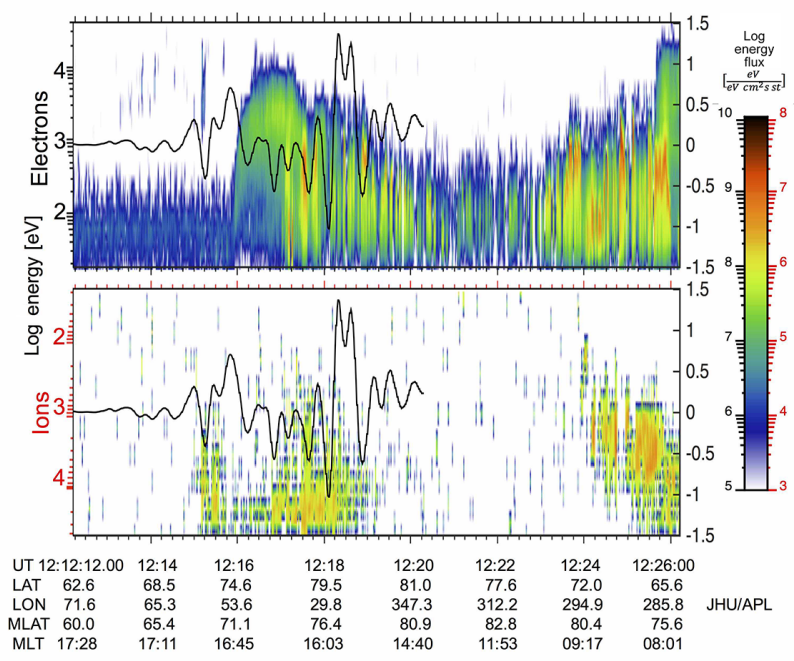

Fig. 9). The quite extreme IMF conditions $\left(B_{y}=-21 \mathrm{nT}\right.$, $B_{z}=20 \mathrm{nT}$ ) are probably responsible for the intense FACs. Adjacent to the auroral oval, Swarm detects a prominent downward FAC, marked HC. From the particle spectrogram, this high-latitude FAC sheet appears at the boundary between auroral oval and polar-rain region, but still on closed field lines. Furthermore, the large negative $B_{y}$ is consistent in both this and the previous case with the larger FAC amplitudes on the duskside. FAC intensities in the morning sector are clearly smaller but distributed over a larger latitudinal range. The positive $B_{x}$ in both cases (see Table 1) infers lobe reconnection in the Southern Hemisphere. As a consequence, more intense FACs would be expected in the south (e.g., Wang et al., 2014). In actual fact, we observe smaller FAC in the Southern Hemisphere (not shown), although the local summer conditions should have favored the intensity. During the previous orbit (see Fig. 8), a downward FAC sheet is detected somewhat detached from the oval on the duskside. Here the significant FAC appears well in the region of polar rain (soft electron precipitation, devoid of ions).

Another class of events is presented in Fig. 10. Here we found two peaks of downward FACs at high latitude. The one at $78^{\circ}$ MLat, adjacent to the auroral oval, appears at the boundary to the polar cap but probably on a closed field line, according to particle signatures. The other more polewardlocated peak ( $83^{\circ}$ MLat) is associated with a narrow isolated Sun-aligned auroral arc. Unfortunately, DMSP was too far away for recording the precipitating particles accompanying the optical feature, but the FUV image shows the fil- 

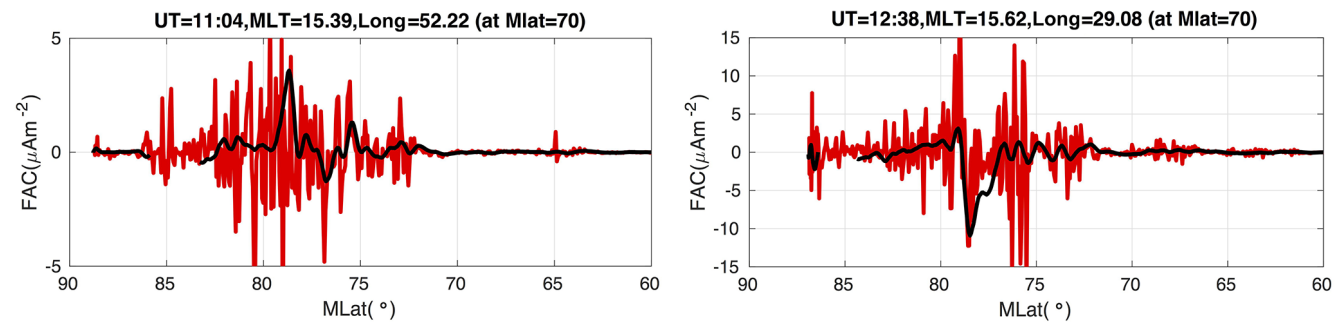

Figure 11. Small-scale FAC activity accompanying the events shown in Figs. 8 and 9. There is clear evidence for filamentary FAC structures at high latitudes.

amentary structure. We still may assume that it was surrounded by polar rain. According to Newell et al. (2009), such clearly detached arcs are rather rare and commonly follow a larger reconfiguration of the IMF orientation. In fact, OMNI data confirmed that a major reorientation of the IMF occurred at 12:23 UT from duskward-northward to dawnward-southward (not shown).

According to our interpretation it seems that, in all six cases, the common feature of the events for which dual FACs do not agree with the individual Swarm $A$ and $C$ results is that these regions tend to occur in the vicinity of precipitating electrons showing evidence of filamentary broadband and/or mono-energetic accelerations or auroral images showing filamentary structures. Moreover, in the particle precipitation data, these regions fall into two categories: (1) near the poleward edge of the auroral oval or (2) in the polar cap. In the first category, studies have found that the poleward region of the auroral oval on the nightside often have filamentary mono-energetic or broadband electron accelerations, which may be associated with Alfvén waves and small-scale currents (e.g., Wing et al., 2013; 2016; Chaston et al., 2002, 2003; Damiano and Johnson, 2012). Newell et al. (1991) developed a classification scheme for the high-latitude particle precipitation. Their boundary plasma sheet (BPS), which is located in the poleward region of the auroral oval, is characterized by electrons having small structures. In fact, this is a key feature that distinguishes BPS from the central plasma sheet (CPS), which is usually found at lower latitude. Some of these filamentary currents may have a component that is parallel to Swarm spacecraft orbits, which may explain the disagreements in the FACs derived from dual and single satellites. In the second category the filamentary electron structures in the polar cap may be associated with polar cap arcs.

In order to check this inference we looked at highresolution FAC estimates from Swarm A. Figure 11 shows the time series of small-scale FACs $(>10 \mathrm{~km}$ horizontal scale) for the two events on 23 December 2014. There is clear evidence for strong bursts of small-scale FACs accompanying the high-latitude FAC events, thus confirming the existence of filamentary current sheets. The lack of prominent small-scale FAC signatures in our Southern Hemi- sphere events can be explained by the small angle between the filament and the satellite track.

The combination of Swarm and DMSP data generally gives a consistent picture of the FAC characteristics observed at high latitude. As more Swarm FAC estimates become available, a comprehensive and statistically significant study of high-latitude current distribution can be performed.

\subsection{Comparison with AMPERE}

Another approach of multi-satellite FAC determination is based on the fleet of Iridium satellites and realized within the AMPERE project (Anderson et al., 2000, 2014). Global distributions of FACs are generated at a $10 \mathrm{~min}$ cadence separately for the Northern And Southern Hemisphere. For two events of prominent high-latitude FACs recorded by Swarm, we compare in Fig. 12 the observations with simultaneous AMPERE FAC maps. In the AMPERE distributions positive values represent upward currents and are shown in red; for convenience, the Swarm convention has been adapted here. The line plots in the top panels of Fig. 12 indicate a qualitative agreement between Swarm and AMPERE results on the dayside, although Swarm FAC estimates had to be low-pass-filtered with a $-3 \mathrm{~dB}$ cutoff period of $90 \mathrm{~s}$ (corresponding to $650 \mathrm{~km}$ wavelength along-track) to make amplitudes comparable. In these two cases Swarm recorded downward FAC at high latitude on the nightside (see blue arrows in top panels). Small circles on the orbit tracks in the lower frames mark the locations of peak current density. However, FAC maps derived from AMPERE data are practically devoid of signal on the nightside. In order to further investigate this apparent discrepancy we had a look at the originally recorded magnetic field deflections by the Iridium satellites. Figure 13 shows 10 min of data centered on the times of our high-latitude events. Just $10 \mathrm{~min}$ are needed by the constellation to cover the auroral region evenly. In these pole-centered maps, noon is at the top. Derived FAC intensities are overplotted on the continents as shaded bands. From these, magnetic latitudes can be estimated in comparison with Fig. 12. Fairly large and consistent magnetic deflections appear on the dayside. On the nightside the magnetic vectors have smaller amplitudes and varying directions. This 

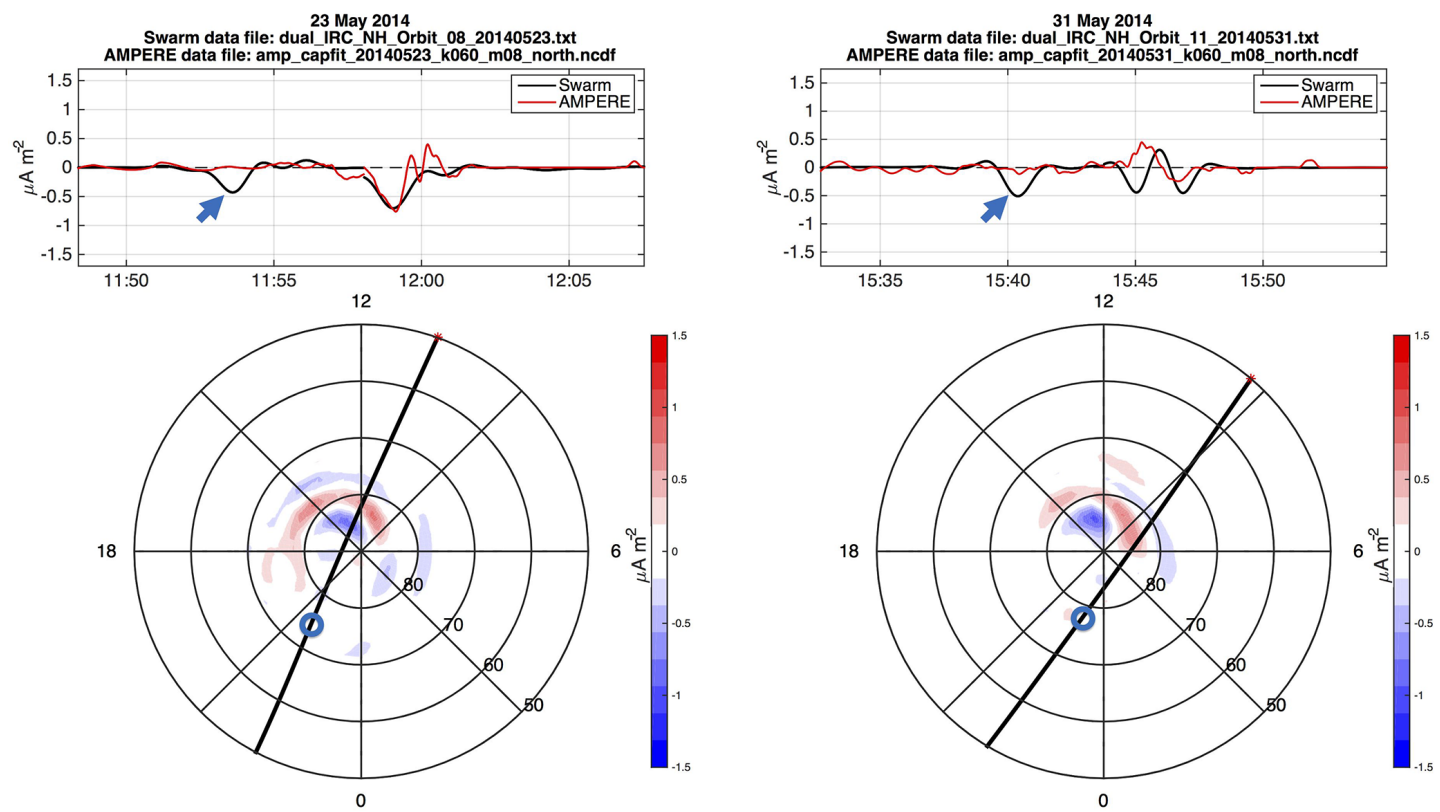

Figure 12. Comparison of Swarm and AMPERE field-aligned current estimates. In the top panels, time series along the Swarm track are compared. Occurrences of high-latitude FACs in Swarm records are marked by arrows in the top and by circles in the bottom plots. The FAC distribution derived from AMPERE is an average over the time 11:58-12:08 UT (left frames) and 15:44-15:54 UT (right frames). Swarm data are adapted to AMPERE conventions; plotted are radial currents (positive outward) low-pass-filtered with a $60 \mathrm{~s}$ cutoff period.

Iridium, 23 May 2014, 12:00 UT

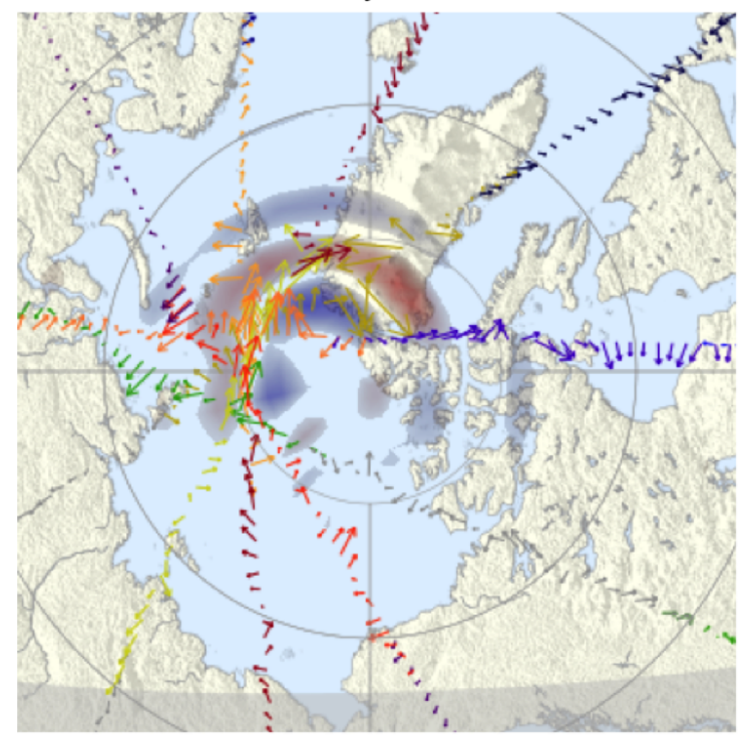

Iridium, 31 May 2014, 15:40 UT

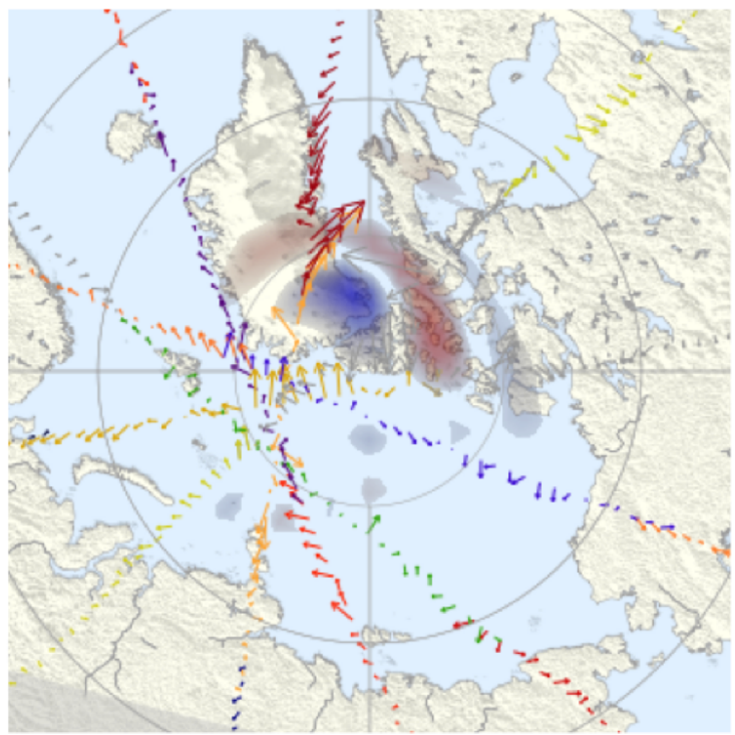

Figure 13. Plots of magnetic field deflection vectors along the tracks of Iridium satellites for the 10 min intervals shown in Fig. 12. Local noon is at the top of the plots. Estimates of FAC sheets are shown as shaded bands like in Fig. 12.

implies that they are caused by smaller-scale FAC structures. The six orbital planes of the Iridium constellation are separated on average by $30^{\circ}$ in longitude (when considering both ascending and descending tracks). In comparison, the pair Swarm A and C is only separated by $1.4^{\circ}$ in longitude. This easily explains why AMPERE results may miss local FAC features. The type of high-latitude FACs during northward IMF, discussed here, obviously falls into that category. 


\section{Summary}

The Swarm constellation of satellites provides the opportunity of improved field-aligned current (FAC) observations. At high latitudes, previous missions have obviously missed a good part of FAC features. This initial study has outlined important characteristics of FAC structures that were not detected by single-satellite approaches. Typical features of these events are as follows:

1. Discrepancies between single- and dual-satellite FAC estimates appear commonly poleward of $75^{\circ}$ MLat. The majority of the events appear on the nightside, where wave activities in the midtail frequently occurs that can couple with the electrons leading to broadband and mono-energetic electrons and FAC filaments (Chaston et al., 2002, 2003; Wing et al., 2013, 2016).

2. Our high-latitude FAC events commonly occur during times of northward IMF. For such conditions magnetic field reconnection poleward of the cusp is expected (Øieroset et al., 1997). Such a configuration is known to favor formation of FACs poleward of the auroral oval.

3. A direct comparison with particle precipitation suggests that the high-latitude FACs are located on closed field lines just adjacent to the polar cap and probably connected to the boundary plasma sheet. (See comments in item 1 on waves in the midtail region causing electron acceleration and FACs.)

4. Auroral images well confirm the presence of filamentary arcs in connection with FACs. From the images a relation to Sun-aligned arcs is suggested.

5. An inspection of the large-scale FACs distribution derived from AMPERE reveals that our high-latitude FACs are not detected. Their scale sizes are obviously too small and thus cannot be resolved by the Iridium satellite constellation. Adjacent tracks are separated by $30^{\circ}$ in longitude.

This initial study has shown that there is a lot of FAC activity at latitudes poleward of the auroral oval during times of northward IMF. A good part of that has probably not been detected by previous missions. The Swarm mission provides the opportunity to improve the situation. In a systematic survey, based on more data, the characteristics of these FAC features should be determined quantitatively.

\section{Data availability}

DMSP SSUSI data products are publicly available at the Johns Hopkins Applied Physics Laboratory (JHU/APL) website http://ssusi.jhuapl.edu/data_products (JHU/APL, 2016b). The DMSP SSJ4/5 data are publicly available from the JHU/APL website: http:
//sd-www.jhuapl.edu/Aurora/dataset_list.html (JHU/APL, 2016a). The Swarm level 2 product "FAC" is freely accessible at https://earth.esa.int/web/guest/swarm/data-access (ESA, 2015).

Acknowledgements. The authors are grateful to Yongliang Zhang for processing auroral images from the SSUSI instrument on DMSP. Simon Wing acknowledges support from the NSF (grant AGS-1058456) and NASA (grants NNX13AE12G, NNX15AJ01G, NNX16AC39G). The European Space Agency (ESA) is acknowledged for providing the Swarm data and for financially supporting the work on developing the Swarm level 2 product "FAC". The authors thank the International Space Science Institute in Bern, Switzerland, for supporting the International Team "Field-Aligned Currents: Their Morphology, Evolution, Source Regions and Generators", on which this study is based. The work of Tao Huang is supported by the National Nature Science Foundation of China (41222030) and the China Scholarship Council (no. 201506270072)

The article processing charges for this open-access publication were covered by a Research Centre of the Helmholtz Association.

The topical editor, G. Balasis, thanks the two anonymous referees for help in evaluating this paper.

\section{References}

Anderson, B. J., Takahashi, K., and Toth, A.: Sensing global Birkeland currents with Iridium $^{\circledR}$ engineering magnetometer data, Geophys. Res. Lett., 27, 4045-4048, doi:10.1029/2000GL000094, 2000.

Anderson, B. J., Korth, H., Waters, C. L., Green, D. L., Merkin, V. G., Barnes, R. J., and Dyrud, L. P.: Development of Large-Scale Birkeland Currents Determined from the Active Magnetosphere and Planetary Electrodynamics Response Experiment (AMPERE), Geophy. Res. Lett., 41, 3017-3025, doi:10.1002/2014GL059941, 2014.

Araki, T., Kamei, T., and Iyemori, T.: Polar cap vertical currents associated with northward interplanetary magnetic field, Geophys. Res. Lett., 11, 23-26, 1984.

Burke, W. J., Kelley, M. C., Sagalyn, R. C., Smiddy, M., and Lai, S. T.: Polar cap electric field structures with a northward interplanetary magnetic field, Geophys. Res. Lett., 6, 21-24, 1979.

Chaston, C. C., Bonnell, J. W., Peticolas, L. M., Carlson, C. W., Mc-Fadden, J. P., and Ergun, R. E.: Driven Alfvén waves and electron acceleration: A FAST case study, Geophys. Res. Lett., 29, 1535, doi:10.1029/2001GL013842, 2002.

Chaston, C. C., Bonnell, J. W., Carlson, C. W., McFadden, J. P., Ergun, R. E., and Strangeway, R. J.: Properties of small-scale Alfvén waves and accelerated electrons from FAST, J. Geophys. Res., 108, 8003, doi:10.1029/2002JA009420, 2003.

Clauer, C. R. and Friis-Christensen, E.: High-latitude dayside electric fields and currents during strong northward interplanetary magnetic field - Observations and model simulation, J. Geophys. Res., 93, 2749-2757, 1988. 
Damiano, P. A. and Johnson, J. R.: Electron acceleration in a geomagnetic Field Line Resonance, Geophys. Res. Lett., 39, L02102, doi:10.1029/2011GL050264, 2012.

Dunlop, M. W., Yang, Y.-Y., Yang, J.-Y., Lühr, H., Shen, C., Olsen, N., Ritter, P., Zhang, Q.-H., Cao, J.-B., Fu, H.-S., and Haagmans, R.: Multi-spacecraft current estimates at Swarm, J. Geophys. Res.-Space, 120, 8307-8316, doi:10.1002/2015JA021707, 2015.

Eriksson, S., Hairston, M. R., Rich, F. J., Korth, H., Zhang, Y., and Anderson, B. J.: High-latitude ionospheric convection and Birkeland current response for the 15 May 2005 magnetic storm recovery phase, J. Geophys. Res., 113, A00A08, doi:10.1029/2008JA013139, 2008.

ESA: European Space Agency, Swarm level 2 product "FAC", available at: https://earth.esa.int/web/guest/swarm/data-access, last access: 17 November 2015.

Fairfield, D. H. and Scudder, J. D.: Polar rain: Solar coronal electrons in the Earth's magnetosphere, J. Geophys. Res., 90, 40554068, 1985.

Fairfield, D. H., Wing, S., Newell, P. T., Ruohoniemi, J. M., Gosling, J. T., and Skoug, R. M.: Polar rain gradients and fieldaligned polar cap potentials, J. Geophys. Res., 113, A10203, doi:10.1029/2008JA013437, 2008.

Finlay, C. C., Olsen, N., and Toeffner-Claussen, L.: DTU candidate field models for IGRF-12 and the CHAOS-5 geomagnetic field model, Earth, Planets, Space, 67, 114, doi:10.1186/s40623-0150274-3, 2015.

Frank, L. A., Craven, J. D., Gurnett, D. A., Shawhan, S. D., Weimar, D. R., Burch, J. L., Winningham, J. D., Chappell, C. R., Waite, J. H., Heelis, R. A., Maynard, N. C., Sugiura, M., Peterson, W. K., and Shelley, E.G.: The theta aurora, J. Geophys. Res., 91, 3177-3224, 1986.

Friis-Christensen, E., Lühr, H., Knudsen, D., and Haagmans, R.: Swarm - An Earth Observation Mission investigating Geospace, Adv. Space Res., 41, 210-216, doi:10.1016/j.asr.2006.10.008, 2008.

Gussenhoven, M. S. and Madden, D.: Monitoring the polar rain over a solar cycle: A polar rain index, J. Geophys. Res., 95, 1039910416, 1990.

Hardy, D. A., Schmitt, L. K., Gussenhoven, M. S., Marshall, F. J., Yeh, H. C., Shumaker, T. L., Hube, A., and Pantazis, J.: Precipitating Electron and Ion Detectors (SSJ/4) for the Block 5D/Flights 6-10 DMSP Satellites: Calibration and Data Presentation, Rep. AFGL-TR-84-0317, Air Force Geophys. Lab., Hanscom Air Force Base, Mass., 1984.

He, M., Vogt, J., Lühr, H., and Sorbalo, E.: Local time resolved dynamics of field-aligned currents and their response to solar wind variability, J. Geophys. Res.-Spaces, 119, 2014JA019776, doi:10.1002/2014JA019776, 2014.

Heelis, R. A.: The effects of interplanetary orientation on dayside high-latitude convection, J. Geophys. Res., 89, 2873-2880, 1984.

Iijima, T. and Shibaji, T.: Global characteristics of northward IMFassociated (NBZ) field-aligned currents, J. Geophys. Res., 92, 2408-2424, 1987.

Iijima, T., Potemra, T. A., Zanetti, L. J., and Bythrow, P. F.: Largescale Birke-land currents in the dayside polar region during strongly northward IMF: Anew Birkeland current system, J. Geophys. Res., 89, 7441-7452, 1984.
JHU/APL: The Johns Hopkins University Applied Physics Laboratory, DMSP SSJ4/5 data, available at: http://sd-www.jhuapl.edu/ Aurora/dataset_list.html, last access: 1 February 2016a.

JHU/APL: The Johns Hopkins University Applied Physics Laboratory, DMSP SSUSI data, available at: http://ssusi.jhuapl.edu/ data_products, last access: 16 February $2016 \mathrm{~b}$.

Korth, H., Anderson, B. J., Frey, H. U., Immel, T. J., and Mende, S. B.: Conditions governing localized high-latitude dayside aurora, Geophys. Res. Lett., 31, L04806, doi:10.1029/2003GL018911, 2004.

Korth, H., Anderson, B. J., Frey, H. U., and Waters, C. L.: Highlatitude electromagnetic and particle energy flux during an event with sustained strongly northward IMF, Ann. Geophys., 23, 1295-1310, doi:10.5194/angeo-23-1295-2005, 2005.

Lühr, H., Park, J., Gjerloev, J. W., Rauberg, J., Michaelis, I., Merayo, J. M. G., and Brauer, P.: Field-aligned Currents' Scale Analysis Performed with the Swarm Constellation, Geophys. Res. Lett., 42, 1-8, doi:10.1002/2014GL062453, 2015a.

Lühr, H., Kervalishvili, G., Michaelis, I., Rauberg, J., Ritter, P., Park, J., Merayo, J. M. G., and Brauer, P.: The inter-hemispheric and F-region dynamo currents revisited with the Swarm constellation, Geophys. Res. Lett., 42, 3069-3075, doi:10.1002/ 2015GL063662, 2015b.

Newell, P. T. and Meng, C.-I.: Creation of theta-auroras: the isolation of plasma sheet fragments in the polar cap, Science, 270, 1338-1341, 1995.

Newell, P. T., Wing, S., Meng, C. I., and Sigillito, V.: The Auroral Oval Position, Structure And Intensity of Precipitation From 1984 Onwards: An Automated On-Line Data Base, J. Geophys. Res., 96, 5877-5882, 1991.

Newell, P. T., Liou, K., and Wilson, G. R.: Polar cap particle precipitation and aurora: Review and commentary, J. Atmos. Sol.-Terr. Phy., 71, 199-215, doi:10.1016/j.jastp.2008.11.004, 2009.

Paxton, L. J., Paxton, D., Morrison, Y., Zhang, H., Kil, B., Wolven, B. S., Ogorzalek, D., Humm, D. C., and Meng, C.-I.: Validation of Remote Sensing Products Produced by the Special Sensor Ultraviolet Scanning Imager (SSUSI) - a Far-UV Imaging Spectrograph on DMSP F16, Proc. in SPIE 4485, Optical Spectroscopic Techniques, Remote Sensing, and Instrumentation for Atmospheric and Space Research IV, 338, doi:10.1117/12.454268, 2002.

Øieroset, M., Sandholt, P. E., Denig, W. F., and Cowley, S. W. H.: Northward interplanetary magnetic field cusp aurora and highlatitude magnetopause reconnection, J. Geophys. Res., 102, 11349_ 11362, doi:10.1029/97JA00559, 1997.

Ritter, P., Lühr, H., and Rauberg, J.: Determining field-aligned currents with the Swarm constellation mission, Earth Planets Space, 65, 1285-1294, doi:10.5047/eps.2013.09.006, 2013.

Ruohoniemi, J. M. and Baker, K. B.: Large-scale imaging of highlatitude convection with super dual auroral radar network HF radar observations, J. Geophys. Res., 103, 20797-20811, 1998.

Vennerstroem, S., Moretto, T., Olsen, N., Friis-Christensen, E., Stampe, A. M., and Watermann, J.: Field-aligned currents in the dayside cusp and polar cap region during northward IMF, J. Geophys. Res., 107, A81188, doi:10.1029/2001JA009162, 2002.

Vennerstroem, S., Moretto, T., Rastätter, L., and Raeder, J.: Field-aligned currents during northward interplanetary magnetic field: Morphology and causes, J. Geophys. Res., 110, A06205, doi:10.1029/2004JA010802, 2005. 
Wang, H., Ridley, A. J., and Lühr, H.: SWMF simulation of field-aligned currents for a varying northward and duskward IMF with nonzero dipole tilt, Ann. Geophys., 26, 1461-1477, doi:10.5194/angeo-26-1461-2008, 2008.

Wang, H., Lühr, H., Shue, J.-H., Frey, H. U., Kervalishvili, G., Huang, T., Cao, X., Pi, G., and Ridley, A. J.: Strong ionospheric field-aligned currents for radial interplanetary magnetic fields, J. Geophys. Res.-Space, 119, 3979-3995, doi:10.1002/2014JA019951, 2014.

Wing, S., Newell, P. T., and Onsager, T. G.: Modeling the entry of magnetosheath electrons into the dayside ionosphere, J. Geophys. Res., 101, 13155-13167, doi:10.1029/96JA00395, 1996.

Wing, S., Newell, P. T., and Ruohoniemi, J. M.: Double cusp: Model prediction and observational verification, J. Geophys. Res., 106, 25571-25593, doi:10.1029/2000JA000402, 2001.
Wing, S., Gkioulidou, M., Johnson, J. R., Newell, P. T., and Wang, C.-P.: Auroral particle precipitation characterized by the substorm cycle, J. Geophys. Res.-Space, 118, 1022-1039, doi:10.1002/jgra.50160, 2013.

Wing, S., Fairfield, D. H., Johnson, J. R., and Ohtani, S.-I.: On the field-aligned electric field in the polar cap, Geophys. Res. Lett., 42, 5090-5099, doi:10.1002/2015GL064229, 2015.

Wing, S., Johnson, J. R., and Camporeale, E.: Dawn-dusk asymmetries in the auroral particle precipitation and their modulations by substorm in: Dawn-Dusk Asymmetry in Planetary Plasma Environments, edited by: Forsyth, C., Haaland, S., and Runov, A., John Wiley \& Sons, Inc, Hoboken, NJ, accepted, 2016. 